\title{
EFEITO DA VELOCIDADE DE ENTRADA EM UM LEITO DE JORRO: MODELAGEM E SIMULAÇÃO
}

\author{
N. A. BONFIM ${ }^{1}$, S. R. F. NETO ${ }^{2}$ \\ ${ }^{1}$ e 2 Universidade Federal de Campina Grande, Centro de Ciências e Tecnologia \\ Unidade Acadêmica de Engenharia Química \\ Laboratório de Pesquisa em Fluidodinâmica e Imagem - LPFI \\ E-mail para contato: natalya.abonfim@gmail.com
}

\begin{abstract}
RESUMO - Aplicou-se a fluidodinâmica computacional (CFD) para avaliar o comportamento de um leito de jorro variando-se os valores da velocidade do gás de alimentação. Empregou-se a abordagem Euleriana-Euleriana para descrever o escoamento heterogêneo de um sistema gás/sólido, adotando-se a Teoria Cinética do Fluxo Granular (KTGF). As simulações foram realizadas utilizando-se o pacote computacional ANSYS FLUENT ${ }^{\circledR}$. Os resultados das simulações indicam que a velocidade do ar de entrada afeta consideravelmente o comportamento fluidodinâmico do leito de jorro, notadamente na altura da fonte de partículas. A modelagem matemática proposta foi capaz de prever satisfatoriamente o comportamento fluidodinâmico do leito de jorro se confrontado com resultados reportados na literatura.
\end{abstract}

\section{INTRODUÇÃO}

O leito de jorro é caracterizado por sua vasta aplicabilidade nos mais variados processos industriais, a exemplo dos processos de secagem, enriquecimento e revestimento de partículas, processos reativos de gaseificação, pirólise, catalíticos entre outros. Tal eficiência se dá pelas altas taxas de transferências de calor e massa que o sistema confere aos processos, e isto decorre dos movimentos cíclicos e contínuos das partículas no interior do equipamento, mantendo a homogeneidade do leito de partículas e favorecendo o contato sólido-fluido. Estudos relacionados à técnica de leito de jorro tem sido alvo de várias pesquisas e trabalhos reportados na literatura, sobretudo em trabalhos experimentais. Observa-se igualmente alguns trabalhos empregando a ferramenta da Fluidodinâmica Computacional (CFD), principalmente para avaliar a fluidodinâmica de leitos de jorro, bem como a eficiência deste sistema dada as suas aplicações. Para isso a modelagem matemática associada à descrição do escoamento multifásico que ocorre no leito de jorro tem como base a Teoria Cinética de Fluxo Granular (KTGF), a qual se expressa através das equações constitutivas associadas a fase granular, ou a fase sólida no caso de um escoamento gás/sólido (ALMEIDA, 2016; BRITTO-COSTA e RUOTOLO, 2015; GOLSHAN, ESGANDARI e ZARGHAMI, 2017; MACHADO et al., 2014).

Zhonghua e Mujumdar (2008) e Du et al. (2006) aplicaram o modelo de dois fluidos (TFM) conjuntamente com o modelo de turbulência e de arraste k-epsilon padrão e Gidaspow, 
respectivamente. Os autores se basearam no trabalho experimental reportado por He et al. (1994a, b). Foi considerado o escoamento ar (fase contínua) e esfera de vidro (fase granular) de $1,41 \mathrm{~mm}$ o diâmetro. Os resultados obtidos pelos autores reproduziram de forma satisfatória o comportamento e a grandeza dos perfis de velocidade obtidos experimentalmente por He et al. (1994a, b).

Com base no exposto, a proposta deste trabalho é apresentar os resultados da influência da velocidade de entrada em um leito de jorro sobre o comportamento fluidodinâmico a partir de diferentes simulações.

\section{METODOLOGIA}

O leito de jorro estudado foi baseado no trabalho de Zhonghua e Mujumdar (2008), cujas dimensões são apresentadas na Figura 1a. A malha numérica foi gerada no ANSYS ICEM $\mathrm{CFD}^{\circledR} 15.0$ adotando-se o critério de discretização sugerido por Duarte (2006), ou seja, o tamanho das arestas ou "edge" devem ficar com valores próximos de 5 a $10 \%$ do valor do diâmetro da partícula. A malha resultante é formada por 22.262 elementos hexaédricos conforme pode ser observado na Figura $1 \mathrm{~b}$.

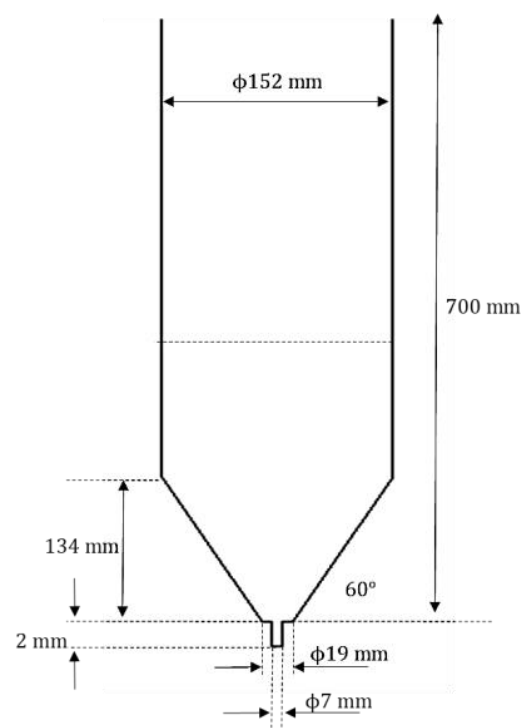

(a)

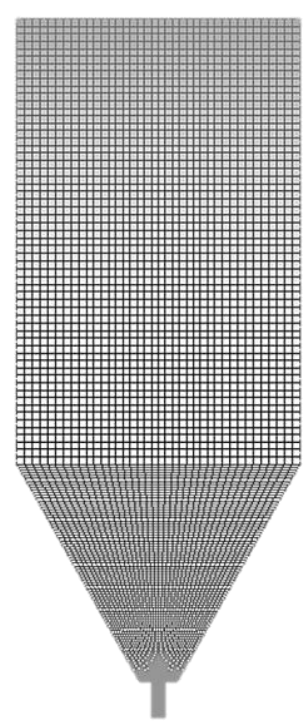

(b)

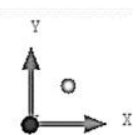

Figura 1 - (a) Dimensões e (b) malha computacional do leito de jorro em estudo.

Para as simulações numérica foi utilizado o ANSYS FLUENT ${ }^{\circledR} 15.0$, adotando-se a abordagem Euleriana-Euleriana para descrever o comportamento do gás (ar) e das partículas (esfera de vidro de $1,41 \mathrm{~mm}$ de diâmetro) em regime transiente para um passo de tempo de $5,0 \times 10^{-4}$ s e um tempo real de simulação de 2,5 s. Foi considerado o modelo da Teoria Cinética do Fluxo Granular (KTGF). Assumiu-se o modelo de arraste "Symmetric" (ANSYS FLUENT THEORY GUIDE, 2015) e o modelo de turbulência k-epsilon "realizable”.

As condições iniciais e de contorno empregadas na resolução do modelo, encontram-se ilustradas na Figura 2. 


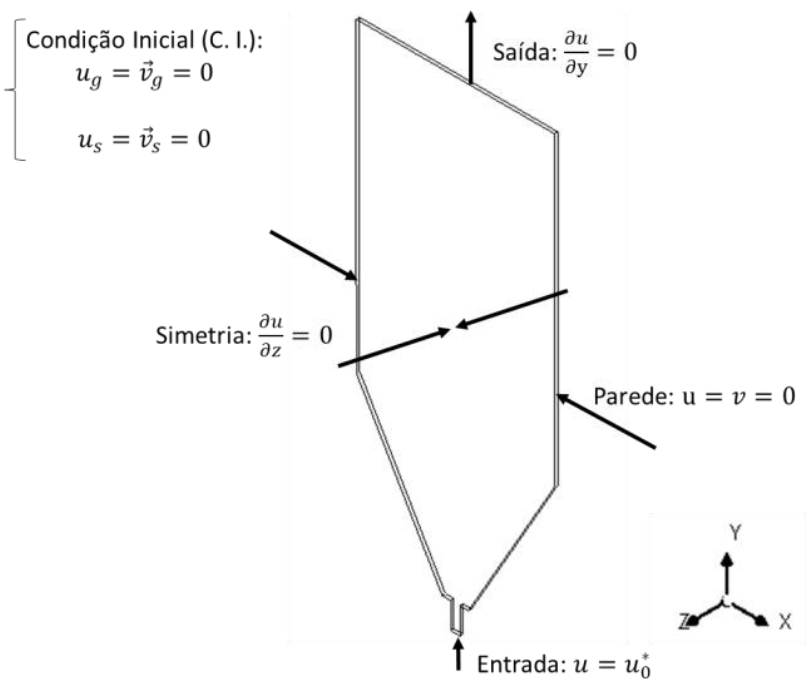

Figura 2 - Condições iniciais e de contorno do caso em estudo.

\section{RESULTADOS}

Nas Figuras 3 e 4 estão representados o campo de fração volumétrica das esferas de vidro (a), o campo vetorial das partículas (b) e os perfis de velocidade das partículas em diferentes posições axiais no leito de jorro (c) para duas velocidades do gás adotada na seção de entrada do leito, 0,6 e 1,25 m.s $\mathrm{s}^{-1}$, respectivamente, para $1 \mathrm{~s}$ de tempo real de simulação.

$$
\begin{aligned}
& 6.104 \mathrm{e}-001 \\
& 5.723 \mathrm{e}-001 \\
& 5.341 \mathrm{e}-001 \\
& 4.960 \mathrm{e}-001 \\
& 4.578 \mathrm{e}-001 \\
& 4.197 \mathrm{e}-001 \\
& 3.815 \mathrm{e}-001 \\
& 3.434 \mathrm{e}-001 \\
& 3.052 \mathrm{e}-001 \\
& 2.671 \mathrm{e}-001 \\
& 2.289 \mathrm{e}-001 \\
& 1.908 \mathrm{e}-001 \\
& 1.526 \mathrm{e}-001 \\
& 1.145 \mathrm{e}-001 \\
& 7.630 \mathrm{e}-002 \\
& 3.815 \mathrm{e}-002 \\
& 0.000 \mathrm{e}+000
\end{aligned}
$$

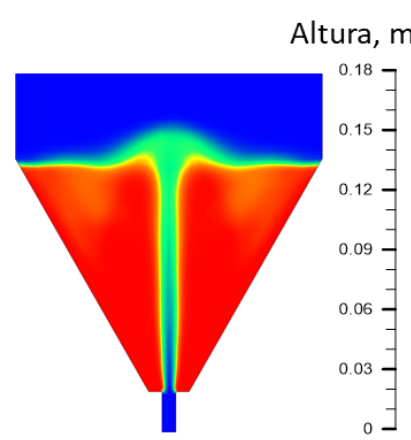

(a)

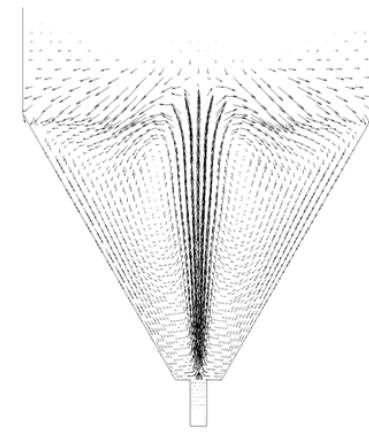

(b)

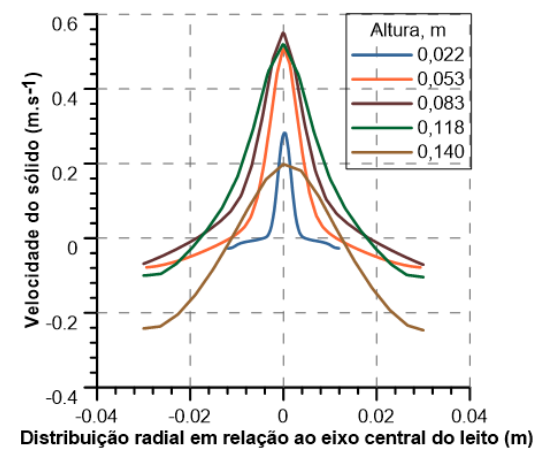

(c)

Figura 3 - (a) Campo de fração volumétrica da partícula, (b) campo vetorial das partículas e (c) perfil de velocidade axial das partículas em diferentes posições no leito, para a velocidade na seção de entrada igual a $0,6 \mathrm{~m} \cdot \mathrm{s}^{-1}$.

Ao observar os campos de fração volumétrica nas Figuras 3a e 4a, é possível identificar as regiões características de um leito de jorro, a região de jorro, situando na parte central do leito, caracterizada pela baixa concentração de partículas; a região anular, situada entre as paredes do leito e a região de jorro, onde se tem a maior concentração de partículas; e por fim, a região de fonte, onde as partículas se comportam como uma fonte onde as partículas ficam 
suspensas pela ação da força de arraste e, ao perder sua intensidade, a força peso as põe sobre o leito na região anular. Observa-se que para a velocidade de $1,25 \mathrm{~m} \cdot \mathrm{s}^{-1}$ a altura que as partículas (esferas de vidro) atingem na região de fonte são maiores do que para $0,6 \mathrm{~m} . \mathrm{s}^{-1}$, o que era esperado tendo em vista uma maior força de arraste proporcionada pela corrente de gás injetada a partir da seção de entrada no leito de jorro. Este comportamento fica mais evidente ao se observar as Figuras 3c e 4c.
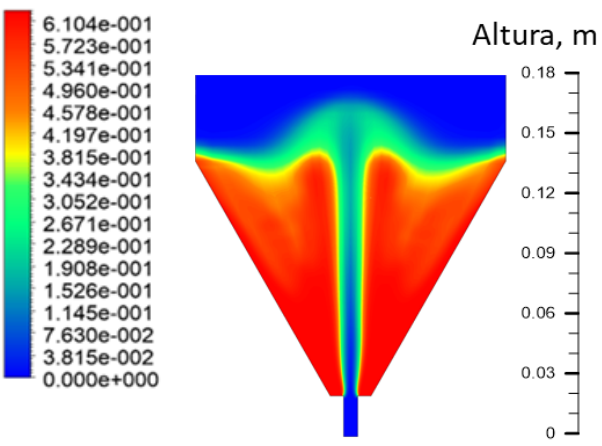

(a)

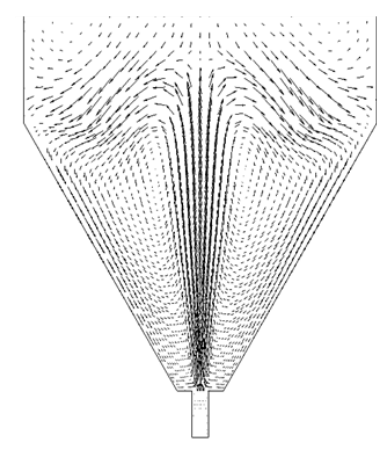

(b)

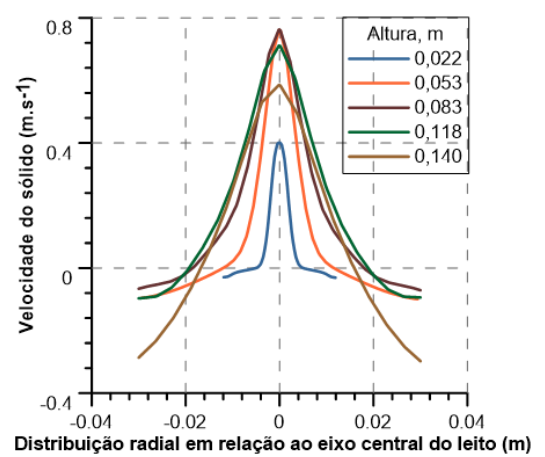

(c)

Figura 4 - (a) Campo de fração volumétrica da partícula, (b) campo vetorial das partículas e (c) perfil de velocidade axial das partículas em diferentes posições no leito, para a velocidade na seção de entrada igual a $1,25 \mathrm{~m} \cdot \mathrm{s}^{-1}$.

A partir das Figuras $3 \mathrm{~b}$ e $4 \mathrm{~b}$, podem ser observadas as disposições das componentes das velocidades no interior do leito de jorro a partir das direções e tamanho dos vetores. É possível identificar variações nos tamanhos dos vetores indicando valores diferentes das componentes de velocidade no leito de jorro, o que ratifica o comportamento das três regiões identificadas no leito de jorro, a exemplo da mudança de direção das partículas ao atingirem a região da fonte, proporcionando zonas de recirculação das partículas. Estas zonas de recirculação podem ser mais pronunciadas com o aumento da velocidade do gás na seção de entrada como pode ser observada na Figura 4b. Comportamento semelhante foi observado por Hosseini, Fattahi e Ahmadi (2015) ao avaliarem a fluidodinâmica de um leito de jorro de partículas esféricas para uma velocidade de entrada de ar de $1,95 \mathrm{~m} . \mathrm{s}^{-1}$.

Nas Figuras 3c e 4c é possível notar que as velocidades das partículas decrescem à medida que se aproxima das paredes do leito, consequência da maior resistência ao movimento das partículas proporcionado pela maior concentração de partículas e as forças atuando entre elas, enquanto que no centro do leito são observadas as maiores velocidades das partículas. Comportamento semelhante das componentes de velocidade destes perfis de velocidade foram obtidos por Zhonghua e Mujumdar (2008) e Du et al. (2006) ao avaliarem a fluidodinâmica de um leito de jorro de partículas esférica assumindo uma velocidade do gás na seção de entrada igual a 0,594 e $0,702 \mathrm{~m} . \mathrm{s}^{-1}$, respectivamente.

\section{CONCLUSÃO}

Os resultados das simulações do leito de jorro de partículas esféricas de vidro possibilitaram concluir que: a) a modelagem matemática empregada descreveu o comportamento fluidodinâmico do leito de jorro, identificando as três regiões reportados em 
vários trabalhos experimentais reportados na literatura; b) a velocidade do gás na seção de entrada tem um papel importante na formação da fonte no leito de jorro; c) a presença das zonas de recirculação das componentes de velocidade das partículas possibilitaram entender e explicar melhor o comportamento fluidodinâmico do leito de jorro.

\section{REFERÊNCIAS}

ALMEIDA, P. S. Análise da fluidodinâmica da casca de cacau em leito de jorro - estudo experimental e simulação via CFD. Dissertação (Mestrado em Energia na área de concentração Multidisciplinar em Energia, Tecnologia e Gestão), Programa de Pós-Graduação em Energia, Universidade Federal do Espírito Santo, São Mateus, 2016.

ANSYS FLUENT THEORY GUIDE. Multiphase Flows, ANSYS, Inc. 2015.

BRITTO-COSTA, P. H., RUOTOLO L. A. M. Eletrorrecuperação de cobre utilizando reator eletroquímico de leito de jorro. Química Nova, v. 38, p. 657-662, 2015.

DU, W., BAO, X., XU, J., WEI, W. Computational fluid dynamics (CFD) modeling of spouted bed: Influence of frictional stress, maximum packing limit and coefficient of restitution of particles. Chemical Engineering Science, Elsevier, v. 61, p. 4558-4570, 2006.

GOLSHAN, S., ESGANDARI, B., ZARGHAMI, R. CFD-DEM and TFM simulation of spouted bed. Chemical Engineering Transactions, AIDIC, v. 57, 2017.

HE, Y.-L., LIM, C. J., GRACE, J. R., ZHU, J.-X. Measurements of voidage profiles in spouted beds. The Canadian Journal of Chemical Engineering, v. 72, p. 229-234, 1994a.

HE, Y.-L., QIN, S.-Z., LIM, C. J., GRACE, J. R. Particle velocity profiles and solid flow patterns in spouted beds. The Canadian Journal of Chemical Engineering, v. 72, p. 561-238, $1994 \mathrm{~b}$.

HOSSEINI, S. H., FATTAHI, M., AHMADI, G. Hydrodynamics studies of a pseudo 2D rectangular spouted bed by CFD. Powder Technology, Elsevier, v. 279, p. 301-309, 2015.

MACHADO, I. P., FERREIRA, A. E. S., MACHADO, A. K. T., DELMIRO, T. M., MEDEIROS, M. F. D. Secagem em leito de jorro da polpa de graviola com adição de leite - Efeito das variáveis de operação sobre a taxa de produção e umidade do pó. In: Anais do XX Congresso Brasileiro de Engenharia Química - COBEQ, Florianópolis, 2014.

ZHONGHUA, W., MUJUMDAR, A. S. CFD modeling of the gas-particle flow behavior in spouted beds. Powder Technology, Elsevier, v. 183, p. 260-272, 2008. 